\title{
Nonlinearity Estimator-Based Control of A Class of Uncertain Nonlinear Systems
}

\author{
Jun Yang, Ting Li, Cunjia Liu, Shihua Li, Wen-Hua Chen
}

\begin{abstract}
The robust control problem of a class of nonlinear systems subject to external disturbances, control gain uncertainty and nonlinear uncertainties is investigated in this paper using a nonlinearity estimator-based control approach. Different from the existing results, the crucial but highly restrictive hypothesis on the boundedness of nonlinear uncertainties is removed from this paper by means of the tools of semi-global stabilization. By delicately constructing a specific composite Lyapunov function for the closed-loop system as well as several useful level sets, the rigorous qualitative robustness performance is presented for the closed-loop system. Finally, an example of a singlelink manipulator is utilized to demonstrate the performance specification claimed by the theoretical analysis.
\end{abstract}

Index Terms-Nonlinearity estimator, control gain uncertainty, qualitative robustness, uncertain nonlinear systems, semi-global stability.

\section{INTRODUCTION}

A fundamental and crucial task of control systems is to deal with external disturbances and uncertainties [1], [2], [3]. The traditional robust control using high control gains is recognized as a major design tool to suppress disturbances/uncertainties in nonlinear control theory [4]. Nevertheless, robust control is mostly achieved at a price of sacrificing the nominal control performance since the control performance in the nominal case is usually not directly taken into account [15]. Such a design philosophy may cause unsatisfactory overall performance since most practical systems usually operate around their nominal operation point, and rarely operate far away from their nominal operation point [5].

As an alternative approach to traditional robust control, various disturbance/uncertainty estimation and attenuation (DUEA) approaches have been proposed for disturbance rejection and uncertainty attenuation [6], [7], [8], [9], [10], [11]. In the context of DUEA, the total uncertainties including external disturbances and plant-model mismatch are usually estimated and compensated online within the closed-loop system [12], [13]. A remarkable merit of DUEA against the traditional robust control is the essential nature of nonworst-case-oriented design. Within the framework of DUEA, an estimator acting as a patch is utilized to online observe the disturbances/uncertainties. In the absence of disturbances/uncertainties, the patch estimator would be inactivated

J. Yang, T. Li and S. Li are with the School of Automation, Southeast University, Key Laboratory of Measurement and Control of CSE, Ministry of Education, Nanjing 210096, China. Corresponding author: Shihua Li, 1sh@seu.edu.cn.

C. Liu and W.-H. Chen are with the Department of Aeronautical and Automotive Engineering, Loughborough University, Leicestershire LE11 3TU, UK. and a baseline controller is able to preserve the nominal control performances. Otherwise, the estimator is activated for uncertainty estimation and compensation, which improves robustness without involving excessive control energy. To conclude, the DUEA framework provides a good balance between the robustness and nominal performance [14], [15].

Note that the DUEA was initially put forward to purely estimate and compensate external disturbances. Later, it has been discovered that DUEA approaches exhibiting promising and powerful ability in estimating and compensating uncertainties caused by plant-model mismatch. Closed-loop stability of DUEA has been thoroughly developed for nonlinear systems subject to various external disturbances [16]. In [12], [13], the total uncertainties including control gain uncertainty and nonlinear uncertainties have been estimated by DUEA, however, few rigorous stability is available when DUEA is used as a robust control method to deal with model uncertainties. Unlike the external disturbance case, the influence of the model uncertainties is actually a function of system states. It is unrealistic to suppose that the uncertainties are bounded for stability analysis. The coupling between the system dynamics and the estimator error dynamics poses great challenges for rigorous robustness stability analysis. In [14], an extended high-gain observer-based control approach was proposed to establish rigorous stability of DUEA with performance recovery. In the field of active disturbance rejection control (ADRC) known as a kind of DUEA approaches, the robustness stability of the closed-loop system under uncertainties is developed by using an observer bandwidth factor evaluation approach [17], [18]. Recently, the work in [19] further established a semiglobal practical stability of ADRC approach for systems with unknown control gain $b$ by using adaptive estimators.

In this paper, a nonlinearity estimator-based control approach is developed for robust control of a class of uncertain nonlinear systems. What is more, we attempt to establish rigorous robustness stability of the resultant closed-loop system. The nominal dynamics of the uncertain nonlinear systems under consideration are nonlinear, which differs from [17], [18], [19]. A preliminary result of this paper has been published in [5], which provides a simple local result. However, to surmount the limitation of local result, it is quite challenging to conduct stability analysis as it is hard to evaluate the bounds of coupling terms between the system dynamics and the estimator error dynamics outside a local region. This issue will be addressed in this paper by means of modifying the tools of semi-global stabilization. To this end, we first construct several interesting level sets which depend on the set of the initial values of both system and observer states. The delicate 
construction of the useful level sets admits their separation from the observer gain factor to be assigned, which opens the door to semi-global stabilization of the closed-loop system in question. It has been shown that the proposed control method guarantees that the system with arbitrary large initial states converges to a prescribed arbitrary small set by assigning large enough observer gain factor. It has been further proved that in the absence of external disturbances, the closed-loop system is semi-global asymptotical stable in the sense that with arbitrary large initial values, the system states will converge to zero by the proposed controller. Finally, a practical example of a single-link manipulator is investigated to demonstrate the theoretical claims presented in the paper. The results show that with certain quantity of uncertainties it is able to tune the observer gain factor such that the stability of the closed-loop systems is guaranteed. The relationship between the observer gain factor and the amount of uncertainties is explicitly demonstrated by the simulation results.

\section{PRELIMINARIES AND PROBLEM FORMULATION}

\section{A. Problem Formulation}

We consider a class of nonlinear systems subject to external disturbances and nonlinear uncertainties, given by

$$
\dot{x}=f(x)+G(x)\left[d_{1}(x) u+d_{2}(x, \omega)\right],
$$

which can be rewritten as follows

$$
\dot{x}=f(x)+G(x)\left[d_{0} u+d(x, \omega, u)\right],
$$

where $x \in \mathbb{R}^{n}$ is the system state vector, $u \in \mathbb{R}$ is the control input, $d(x, \omega, u)=\left(d_{1}(x)-d_{0}\right) u+d_{2}(x, \omega) \in \mathbb{R}$ denotes the lumped uncertainty including both external disturbances and model uncertainties, $d_{1}(x)$ is an unknown control coefficient whose known nominal part is $d_{0} \neq 0$ that is a constant, $d_{2}(x, \omega)$ is an unknown function denoting the uncertain effects caused by nonlinear uncertainties and external disturbances, $\omega$ is the external disturbance satisfying that $\omega$ and $\dot{\omega}$ are defined on the compact set $\mathcal{D} \subset \mathbb{R}^{q} . f(x)$ and $G(x)$ are known smooth nonlinear functions with $\|G(x)\| \neq 0$ for any $x \in \mathbb{R}^{n}$ where $\|\cdot\|$ denotes the Euclidean norm. Suppose that the lumped uncertainty $d(x, \omega, u)$ satisfies the following assumption.

Assumption 1: The lumped uncertainty $d(x, \omega, u)$ is partially continuously differentiable in terms of the arguments $x$ and $\omega$, and $\left|d_{1}(x)\right| \neq 0$ for any $x \in \mathbb{R}^{n}$. Moreover, there exists a known positive constant $\delta_{0}<1$ and known nonnegative continuous scalar functions $\bar{\delta}_{1}(x)$ and $\bar{\delta}_{2}(x)$ such that

$$
\begin{aligned}
& \left|\frac{d_{1}(x)-d_{0}}{d_{0}}\right| \leq \delta_{0}, \\
& \left\|\frac{\partial d_{2}}{\partial x}\right\| \leq \bar{\delta}_{1}(x), \quad\left\|\frac{\partial d_{2}}{\partial \omega}\right\| \leq \bar{\delta}_{2}(x),
\end{aligned}
$$

for any $x \in \mathbb{R}^{n}$ and $\omega \in \mathcal{D}$.

Remark 1: The lumped uncertainty $d(x, \omega, u)$ is unknown which could include control coefficient uncertainty, external disturbances and model uncertainties including unmodelled nonlinear dynamics, parametric perturbations, as well as other static structured/unstructured modeling errors. The assumptions on the lumped uncertainties are quite different between that in this paper and the existing ones. In most of existing works on DUEA, it is generally required that both the lumped uncertainty and its derivative with respect to time are bounded by some constants, that is, $|d(x, \omega, u)|<c_{1}$ and $|\dot{d}(x, \omega, u)|<c_{2}$. This is quite restrictive because $d(x, \omega, u)$ and $\dot{d}(x, \omega, u)$, which are functions in terms of their arguments $x, \omega$ and $u$, may violate the restrictions $|d(x, \omega, u)|<c_{1}$ and $|\dot{d}(x, \omega, u)|<c_{2}$ as $x$ and $u$ go toward infinity. For a simple example that $d(x, \omega, u)=x$, the condition $|d(x, \omega, u)|<c_{1}$ means that $x<c_{1}$, which implies that the state $x$ is always bounded and stable regardless of the control. Based on this statement, the existing works can only obtain local stability result. However, in this paper, the assumption on the lumped uncertainties is supposed to be bounded by some nonlinear functions, that is, $\left\|\frac{\partial d_{2}}{\partial x}\right\| \leq \bar{\delta}_{1}(x)$ and $\left\|\frac{\partial d_{2}}{\partial \omega}\right\| \leq \bar{\delta}_{2}(x)$. Under the framework of semi-global stabilization, we only require that the initial values of the states are within a compact set, while the real states can go beyond this compact set. Since the lumped uncertainty are functions in terms of system states, they generally can not be bounded by a fixed compact set. This imposes great challenges for the design and analysis of the closed-loop system under the proposed control approach in the context of semi-global stabilization rather than local one.

Remark 2: The hypothesis $\left|\left(d_{1}(x)-d_{0}\right) / d_{0}\right| \leq \delta_{0}$ in Assumption 1 (which indicates that $d_{1}(x)$ and $d_{0}$ must have the same signs) should be taken into account for theoretical proof of the paper. However, the nominal value of $d_{0}$ generally can be chosen in a wide range such that the hypothesis holds. Actually, for any unknown control gain satisfying $C_{1}<d_{1}(x)<C_{2}$ (or $-C_{2}<d_{1}(x)<-C_{1}$ ), where $C_{1}$ and $C_{2}$ are arbitrary positive constants. By selecting $d_{0}>C_{2} / 2$ (or $d_{0}<-C_{2} / 2$ ), the condition $\left|\left(d_{1}(x)-d_{0}\right) / d_{0}\right| \leq \delta_{0}$ is satisfied.

Assumption 2: Suppose that the equilibrium $x=0$ of the nominal system of (2), i.e., $\dot{x}=f(x)+d_{0} G(x) u$ is globally asymptotically stabilized by a static state feedback controller $\bar{u}(x)=\alpha(x)$, where $\alpha(x)$ is continuously differentiable and can be designed by some nonlinear control method. That is, the closed-loop nominal system under the baseline controller $\bar{u}(x)$, governed by $\dot{x}=f_{c}(x)$ is globally asymptotically stable with $f_{c}(x)=f(x)+d_{0} G(x) \alpha(x)$.

The objective of this paper is to develop a nonlinearity estimator-based control approach for nonlinear system (2) such that the equilibrium of the closed-loop system is semi-globally stable even in the presence of nonlinear uncertainties and external disturbances.

\section{B. Preliminaries}

We list some crucial definitions and lemmas for synthesis and analysis of the main result of the paper.

Definition 1: ${ }^{[20]}$ A point $x=0$ of system $\dot{x}=f(x, u)$ is said to be semi-globally practically stabilizable by dynamic state feedback if, for each pair of compact sets $\left(X_{s}, X_{l}\right)$ that are the neighborhoods of $(0,0)$ and satisfy $X_{s} \subset X_{l}$, there exists a locally Lipschitz dynamic state feedback $u=$ $h_{1}(x, \xi), \dot{\xi}=h_{2}(x, \xi)$ and a pair of compact sets $\left(X_{\xi, s}, X_{\xi, l}\right)$ such that all the solutions of the closed-loop system starting 
from the set $X_{l} \times X_{\xi, l}$ are captured by the set $X_{s} \times X_{\xi, s}$. If the set $X_{s} \times X_{\xi, s}=\{(0,0)\}$, the point $x=0$ is said to be semi-globally stabilizable by dynamic state feedback.

Lemma $1^{[21]}$ For given real numbers $m>0, n>0$ and $a$, there exists $c>0$ such that

$\left|a x^{m} y^{n}\right| \leq c|x|^{m+n}+\frac{n}{m+n}\left(\frac{m}{(m+n) c}\right)^{\frac{m}{n}}|a|^{\frac{m+n}{n}}|y|^{m+n}$,

for all $x \in \mathbb{R}$ and $y \in \mathbb{R}$.

Lemma $2^{[13]}$ Suppose that a continuously differentiable function $F(x, y, z) \in \mathbb{R}^{p}$ satisfies

$$
|\mathfrak{h}|\left\|\frac{\partial F}{\partial z}\right\| \leq \epsilon, \quad \forall(x, y, z) \in \mathbb{R}^{n} \times \mathbb{R}^{m} \times \mathbb{R}^{p},
$$

where $0<\epsilon<1$ is a positive constant, $\mathfrak{h}$ is a constant. Then for any function $\operatorname{sat}(\cdot)$ whose derivative is dominated by one, i.e.

$$
\left\|\frac{\partial \mathrm{sat}}{\partial s}(s)\right\| \leq 1, \forall s \in \mathbb{R}^{p},
$$

there exists a unique continuously differentiable function $\Phi_{\text {sat }}(\theta, \varrho, x, y)$ solving the equation

$$
z=\theta-\mathfrak{h} \operatorname{sat}(F(x, y, z)-\varrho),
$$

where $\theta \in \mathbb{R}^{p}$ and $\varrho \in \mathbb{R}^{p}$.

Lemma $3^{[1]}$ Suppose that the equilibrium $x=0$ of the system

$$
\dot{x}=H(x)
$$

is globally asymptotically stable. Then there exists a $\mathcal{C}^{1}$ function $V: \mathbb{R}^{n} \rightarrow \mathbb{R}_{\geq 0}$ such that

$$
\begin{aligned}
\alpha_{1}(\|x\|) & \leq V(x) \leq \alpha_{2}(\|x\|), \\
\frac{\partial V}{\partial x} H(x) & \leq-\Phi(x),
\end{aligned}
$$

where $\alpha_{1}, \alpha_{2}$ and $\Phi$ are continuous positive definite and class $\mathcal{K}_{\infty}$ functions

Lemma $4^{[1]}$ Let $x=0$ be an equilibrium point for the nonlinear system $\dot{x}=f(x)$, where $f: \mathcal{D}_{0} \rightarrow \mathbb{R}^{n}$ is continuously differentiable, $\mathcal{D}_{0}=\left\{x \in \mathbb{R}^{n} \mid\|x\|<r_{0}\right\}$, and $\frac{\partial f(x)}{\partial x}$ is bounded on $\mathcal{D}_{0}$. If the equilibrium $x=0$ is locally exponentially stable; that is, the trajectories of the system satisfy

$$
\|x(t)\| \leq k\left\|x\left(t_{0}\right)\right\| e^{-\lambda\left(t-t_{0}\right)}, \quad \forall x\left(t_{0}\right) \in \mathcal{D}_{1}, \quad \forall t \geq t_{0} \geq 0,
$$

where $\lambda$ is a positive constant, $\mathcal{D}_{1}=\left\{x \in \mathbb{R}^{n} \mid\|x\|<r_{1}\right\}$, and $r_{1}$ is a positive constant satisfying $r_{1} \leq r_{0} / k$ with $k>0$. Then, there is a $\mathcal{C}_{1}$ function $V: \mathcal{D}_{1} \rightarrow \mathbb{R}$ such that

$$
\begin{aligned}
c_{1}\|x\|^{2} \leq V(x) & \leq c_{2}\|x\|^{2}, \\
\frac{\partial V}{\partial x} f(x) & \leq-c_{3}\|x\|^{2}, \\
\left\|\frac{\partial V}{\partial x}\right\| & \leq c_{4}\|x\|,
\end{aligned}
$$

for some positive constants $c_{1}, c_{2}, c_{3}$, and $c_{4}$.

\section{MAIN RESUlts}

\section{A. Nonlinearity Estimator-Based Control Design}

To begin with, a nonlinearity estimator is designed below to estimate the lumped uncertainties $d(x, u, \omega)$ in system (2), which is depicted by

$$
\begin{aligned}
& \dot{\xi}=-\operatorname{Lh}(x)\left[f(x)+G(x)\left(d_{0} u+\xi+p(x)\right)\right], \\
& \hat{d}=\xi+p(x), \frac{\partial p(x)}{\partial x}=\operatorname{Lh}(x),
\end{aligned}
$$

where $\xi \in \mathbb{R}$ is the internal state of the observer, $\hat{d} \in \mathbb{R}$ is the estimate of the lumped uncertainty $d(x, \omega, u), L$ is a positive observer gain factor to be designed, and $h(x)$ is the continuous observer gain which is regardless of the scalar $L$. The error vector of the nonlinearity estimator (8) is defined as follows

$$
\zeta=d-\hat{d} .
$$

With the condition given in Assumption 2, one can obtain from Lemma 3 that there exist continuous positive definite and $\mathcal{K}_{\infty}$ functions $\underline{\alpha}, \bar{\alpha}$, and $\phi$, and a $\mathcal{C}^{1}$ function $V: \mathbb{R}^{n} \rightarrow \mathbb{R}_{\geq 0}$ satisfying

$$
\begin{aligned}
\underline{\alpha}(\|x\|) \leq V(x) & \leq \bar{\alpha}(\|x\|), \\
\frac{\partial V}{\partial x} f_{c}(x) & \leq-\phi(x) .
\end{aligned}
$$

Define a $n$-dimensional super cube set as $\Gamma_{r}^{n} \triangleq[-r, r]^{n}$. Let $C=\max \left\{V(x) \mid x \in \Gamma_{r}^{n}\right\}$. We then define the level sets $\Delta_{C}$ and $\Delta$ as follows

$$
\begin{aligned}
& \Delta_{C}=\left\{x \in \mathbb{R}^{n}: V(x) \leq C\right\} \\
& \Delta=\left\{(x, \zeta, \omega) \in \mathbb{R}^{n} \times \mathbb{R} \times \mathbb{R}^{q}: V(x) \leq C+1,|\zeta| \leq 1, \omega \in D\right\} .
\end{aligned}
$$

Similarly we can construct a level set $\Delta_{C+1}$. By construction we have $\Gamma_{r}^{n} \subset \Delta_{C} \subset \Delta_{C+1}$. As per the above construction, the nonlinearity estimator-based control law is designed as follows

$$
u=\alpha(x)-\operatorname{SAT}_{M}(\hat{d}) / d_{0},
$$

where $M=\sup _{(x, \zeta, \omega) \in \Delta} \mid\left(\left(d_{1}(x)-d_{0}\right) \alpha(x)+d_{2}(x, \omega)-\right.$ $\zeta) d_{0} / d_{1} \mid, \operatorname{SAT}_{M}(\cdot)$ is an odd function defined by

$\operatorname{SAT}_{M}(s)= \begin{cases}s, & \text { if } 0 \leq s \leq M \\ (s-M)(1+M-s)+M, & \text { if } M<s<M+0.5 \\ M+0.25, & \text { if } s \geq 0.5+M\end{cases}$

The function $\operatorname{SAT}_{M}(\cdot)$ is nondecreasing, continuously differentiable with a locally Lipschitz derivative, bounded uniformly in $M$, and its derivative satisfies $0 \leq \frac{\mathrm{dsAT}_{M}}{\mathrm{~d} s} \leq 1$.

\section{B. Stability Analysis}

Thanks to Lemma 2, the control law (12) can be rewritten as

$$
u=\Xi(\alpha(x), \zeta, x, \omega),
$$

and $\Xi(\cdot)$ is a continuously differentiable function. Denote $d_{\Xi}(x, \omega, \zeta) \triangleq d(x, \omega, \Xi(\alpha(x), \zeta, x, \omega))$. Substituting the proposed control law (14) with the nonlinearity estimator (8) into 
the nonlinear system (2), one obtains the closed-loop system below

$$
\begin{aligned}
& \dot{x}=f_{c}(x)+G(x)\left(d_{\Xi}(x, \omega, \zeta)-\operatorname{SAT}_{M}\left(d_{\Xi}(x, \omega, \zeta)-\zeta\right)\right), \\
& \dot{\zeta}=-\operatorname{Lh}(x) G(x) \zeta+\dot{d}_{\Xi}(x, \omega, \zeta),
\end{aligned}
$$

where

$$
\dot{d}_{\Xi}=\frac{\partial d_{\Xi}}{\partial x} \dot{x}+\frac{\partial d_{\Xi}}{\partial \zeta} \dot{\zeta}+\frac{\partial d_{\Xi}}{\partial \omega} \dot{\omega}
$$

Note that

$$
\begin{aligned}
\frac{\partial d_{\Xi}}{\partial x} & =\left(1+\frac{1}{d_{0}} \frac{\partial d}{\partial u} \frac{\partial \mathrm{SAT}_{M}}{\partial s}\right)^{-1}\left(\frac{\partial d}{\partial x}+\frac{\partial d}{\partial u} \frac{\partial \alpha}{\partial x}\right), \\
\frac{\partial d_{\Xi}}{\partial \zeta} & =\left(1+\frac{1}{d_{0}} \frac{\partial d}{\partial u} \frac{\partial \mathrm{SAT}_{M}}{\partial s}\right)^{-1} \frac{1}{d_{0}} \frac{\partial d}{\partial u} \frac{\partial \mathrm{SAT}_{M}}{\partial s}, \\
\frac{\partial d_{\Xi}}{\partial \omega} & =\frac{\partial d_{2}}{\partial \omega} .
\end{aligned}
$$

Inserting (17) into (15), one obtains the closed-loop system having the following form

$$
\begin{aligned}
\dot{x}= & f_{c}(x)+G(x)\left(d_{\Xi}(x, \omega, \zeta)-\operatorname{SAT}_{M}\left(d_{\Xi}(x, \omega, \zeta)-\zeta\right)\right), \\
\dot{\zeta}= & -L\left(1+\frac{1}{d_{0}} \frac{\partial d}{\partial u} \frac{\partial \operatorname{SAT}_{M}}{\partial s}\right) h(x) G(x) \zeta+\psi_{1}(x, \zeta, \omega) \\
& +\psi_{2}(x, \zeta, \omega, \dot{\omega})+\psi_{3}(x, \zeta, \omega)\left(d_{\Xi}(x, \omega, \zeta)\right. \\
& \left.-\operatorname{SAT}_{M}\left(d_{\Xi}(x, \omega, \zeta)-\zeta\right)\right) .
\end{aligned}
$$

with

$$
\begin{aligned}
\psi_{1}(x, \zeta, \omega) & =\left(\frac{\partial d}{\partial x}+\frac{\partial d}{\partial u} \frac{\partial \alpha}{\partial x}\right) f_{c}(x), \\
\psi_{2}(x, \zeta, \omega, \dot{\omega}) & =\left(1+\frac{1}{d_{0}} \frac{\partial d}{\partial u} \frac{\partial \operatorname{SAT}_{M}}{\partial s}\right) \frac{\partial d_{2}}{\partial \omega} \dot{\omega}, \\
\psi_{3}(x, \zeta, \omega) & =\left(\frac{\partial d}{\partial x}+\frac{\partial d}{\partial u} \frac{\partial \alpha}{\partial x}\right) G(x) .
\end{aligned}
$$

For convenient of expression, we further define a super cube set $\Pi_{x}$ and a super compact set $\Pi_{\xi}$ as $\Pi_{x} \triangleq \Gamma_{r}^{n}=[-r, r]^{n}$ and $\Pi_{\xi} \triangleq \Gamma_{r}=[-r, r]$, respectively. The semi-global practical stability of the closed-loop system (18) is given by the following theorem.

Theorem 1: Suppose that Assumptions 1 and 2 are satisfied for the nonlinear system (2). Given any arbitrarily large number $r>0$ and any arbitrarily small number $\varepsilon>0$, there exist a positive scalar $L$ and a finite time $T_{f}>0$, such that, for $\forall t \geq T_{f}$, all the solutions of the closed-loop system (2)(8)-(12), with initial states in $\Pi_{x} \times \Pi_{\xi}$, are captured by the set $\bar{Q}_{\varepsilon}^{n} \times \bar{Q}_{\varepsilon} \triangleq\left\{x \in \mathbb{R}^{n}:\|x\| \leq \varepsilon\right\} \times\{\zeta \in \mathbb{R}:|\zeta| \leq \varepsilon\}$ if the observer gain $h(x)$ is selected such that $h(x) G(x)>0$ for any $x \in \mathbb{R}^{n}$.

Proof. The proof of the theorem is divided into two steps. For the first one, we prove that the solution of the closed-loop system is well defined on $[0, \infty)$ and furthermore the system states will enter the set $\Delta$ in finite time and never escape once enter. Then, it is proved that the trajectories of the closed-loop system will be captured by an arbitrarily small set $\bar{Q}_{\varepsilon}^{n} \times \bar{Q}_{\varepsilon}$.

Step 1. There exist a positive scalar $L$ and a finite time $T>0$ such that the trajectories of the closed-loop system (8)(12)-(18) enter the set $\Delta$ and remain therein for all $t \geq T$.
Keeping the estimator (8) and error definition (9) in mind, it follows from $(x(0), \xi(0)) \in \Pi_{x} \times \Pi_{\xi}$ that the initial condition satisfies

$$
V(x(0)) \leq C \quad \text { and } \quad|\zeta(0)| \leq b(1+L),
$$

where $b$ is a positive constant depending on $r$.

Now consider any solution of (18) defined on the open set $\Lambda \triangleq\{(x, \zeta): V(x) \leq C+1, \zeta \in \mathbb{R}\}$ with the initial condition satisfying (20). Then, we conclude that there exists a positive scalar $L$ such that a solution of (18) is well defined on a right maximal interval $\left[0, T_{c}\right)$ with $T_{c}=\infty$. We prove the above conclusion by contradiction. Toward that end, we suppose that for $\forall L \in \mathbb{R}, T_{c}$ were finite. As the fact that $\zeta \in \mathbb{R}$ for $\forall L \in \mathbb{R}$ and $\forall t \in\left[0, T_{c}\right)$ whether $T_{c}$ is finite or $\infty$, we know $\lim _{t \rightarrow T_{c}} V(x(t))=C+1$. Then, from (10), (18) and the boundedness of $\operatorname{SAT}_{M}(\cdot)$, we know that the functions $\Xi(\cdot)$ and $d_{\Xi}(\cdot)$ are bounded functions. Hence, there exists a positive constant $\eta_{1}$ (independent of $L$ ) such that, for $\forall(x, \zeta) \in \Lambda$,

$$
\begin{aligned}
\dot{V} & \leq-\phi(x)+\left|\frac{\partial V}{\partial x}\left(G(x)\left(d_{\Xi}(x, \omega, \zeta)-\operatorname{SAT}_{M}\left(d_{\Xi}(x, \omega, \zeta)-\zeta\right)\right)\right)\right| \\
& \leq \eta_{1} .
\end{aligned}
$$

Denote $T_{1}=\frac{1}{2 \eta_{1}}$. From (20) and (21), we know that

$$
V(x(t)) \leq V(x(0))+\eta_{1} t \leq C+\frac{1}{2}, \quad \forall t \in\left[0, T_{1}\right],
$$

which implies that $T_{c}>T_{1}$. It follows from Assumption 1 that, for $\forall(x, \zeta) \in \Lambda$,

$$
1+\frac{1}{d_{0}} \frac{\partial d}{\partial u} \frac{\partial \mathrm{SAT}_{M}}{\partial s} \geq 1-\left|\frac{d_{1}(x)-d_{0}}{d_{0}}\right| \geq 1-\delta_{0} .
$$

Therefore, by Lemma 1 and (18), one obtains that there are three positive real numbers $\eta_{0}, \eta_{2}$ and $\eta_{3}$ (independent of $L$ ) such that

$$
\frac{\mathrm{d} \zeta^{2}}{\mathrm{~d} t} \leq-2\left(L-\eta_{2}\right) \eta_{0} \zeta^{2}+\eta_{3}, \quad \forall(x, \zeta) \in \Lambda .
$$

Then it follows from (20) and (24) that, for $\forall t \in\left[0, T_{c}\right.$ ),

$$
|\zeta(t)| \leq b(1+L) e^{-\left(L-\eta_{2}\right) \eta_{0} t}+\sqrt{\frac{\eta_{3}}{2\left(L-\eta_{2}\right) \eta_{0}}} .
$$

To this end, there exists a positive constant $L_{1}$ such that, for $L \geq L_{1}$

$$
|\zeta(t)| \leq \min \left\{1, \frac{1}{2 \eta_{4} T_{c}}\right\}, \quad \forall t \in\left[T_{1}, T_{c}\right),
$$

where $\eta_{4}$ is a positive constant ensuring

$$
\dot{V} \leq-\phi(x)+\eta_{4}|\zeta|, \quad \forall(x, \zeta, \omega) \in \Delta .
$$

It follows from (20), (26) and (27) that

$$
V(x(t)) \leq V(x(0))+\eta_{4}|\zeta| T_{c} \leq C+\frac{1}{2}, \quad \forall t \in\left[T_{1}, T_{c}\right),
$$

which is a contradiction. Thus, we can find a positive scalar $L$ to ensure that $T_{c}=\infty$ and the closed-loop solution is bounded and remains in the set $\Lambda$. Then, by (24) and (25), we obtain 
that there exist a positive constant $L_{2}$ and a finite time $T$ such that, for $L \geq L_{2}$,

$$
|\zeta(t)| \leq 1, \quad \forall t \in[T, \infty),
$$

which implies that the trajectories of the closed-loop system enter the set $\Delta$ and remain therein for all $t \geq T$.

Step 2. The trajectories of the closed-loop system will finally enter an arbitrarily small set $\bar{Q}_{\varepsilon}^{n} \times \bar{Q}_{\varepsilon}$. By the previous argument in Step 1, we know that when $L \geq L_{2}$, the trajectories of the closed-loop system enter the set $\Delta$ and remain therein for all $t \geq T$. Therefore, we only need to consider the case that $(x, \zeta, \omega) \in \Delta$ for $t \geq T$ and $L \geq L_{2}$.

Define a Lyapunov function for the closed-loop system as $W(x, \zeta)=V(x)+U(\zeta)$ with $U(\zeta)=\frac{1}{2} \zeta^{2}$. For any given $\varepsilon>0$, there exists a constant $\rho \in(0,1)$ such that

$$
\Omega_{\rho} \subset \bar{Q}_{\varepsilon}^{n} \times \bar{Q}_{\varepsilon},
$$

where $\Omega_{\rho}$ is a compact level set defined by $\Omega_{\rho} \triangleq$ $\{(x, \zeta) \in \Lambda: W(x, \zeta) \leq \rho\}$. To facilitate the following analysis, we further define two sets as follows

$$
\begin{aligned}
& S_{1}=\{(x, \zeta) \in \Lambda: \rho \leq W(x, \zeta)\}, \\
& S_{2}=\{(x, \zeta): V(x) \leq C+1, \quad U(\zeta) \leq 1\} \cap S_{1} .
\end{aligned}
$$

Define a compact set $S_{0}=S_{2} \cap\{(x, \zeta): \zeta=0\}$. It then can be derived from the construction of $S_{2}$ and $\zeta=0$ for $(x, \zeta) \in S_{0}$ that $x \neq 0$ for $(x, \zeta) \in S_{0}$. As such, it follows from Assumption 2 and Lemma 3 that

$$
\dot{W}(x, \zeta) \leq-\phi(x)<0, \forall(x, \zeta) \in S_{0} .
$$

By continuity, $\dot{W}(x, \zeta)$ is negative at each point of some open set $S_{0}^{-} \supset S_{0}$. We then consider the compact set $\bar{S}_{0}=S_{2} \backslash S_{0}^{-}$. Since $\zeta \neq 0$ at each point of $\bar{S}_{0}$, there exist two constants $\bar{e}>\underline{e}>0$ such that $\underline{e} \leq|\zeta| \leq \bar{e}$ for $(x, \zeta) \in \bar{S}_{0}$.

Then for all $(x, \zeta) \in \bar{S}_{0}, \dot{W}(x, \zeta)$ can be estimated from (18) and given by

$$
\dot{W}(x, \zeta) \leq-\phi(x)+\eta_{4} \bar{e}-\left(L-\eta_{2}\right) \underline{e}^{2}+\frac{\eta_{3}}{2} .
$$

By (32), one can get a constant $L_{3}>0$ such that, for $L>$ $L^{*}=\max \left\{L_{2}, L_{3}\right\}, \dot{W}(x, \zeta)<0$ at each point of $S_{2}$.

Invoking the statements in both Step 1 and Step 2, it can be concluded that there exists the time $T_{f}>0$ such that, for $t \geq T_{f}$ and $L>L^{*}$, the trajectories of the closed-loop will enter the set $\Omega_{\rho} \subset \bar{Q}_{\varepsilon}^{n} \times \bar{Q}_{\varepsilon}$.

In the following, we further establish the semi-global asymptotical stability of the closed-loop systems without the external disturbances.

Theorem 2: Suppose that Assumptions 1 and 2 are satisfied for the nonlinear system (2) without the external disturbances (e.g., $\omega=0), d(x, u)$ is twice partially continuously differentiable on $x$, and the equilibrium $x=0$ for $\dot{x}=f_{c}(x)$ is locally exponentially stable. Given any arbitrarily large number $r>0$, there exists a positive scalar $L$ such that all the solutions of the closed-loop system (2)-(8)-(12), with initial states in $\Pi_{x} \times \Pi_{\xi}$, will converge to the origin if the observer gain $h(x)$ is selected such that $h(x) G(x)>0$ for any $x \in \mathbb{R}^{n}$.

Proof. The proof of this theorem can be proved by firstly addressing the semi-global practical stability, and thereafter the locally asymptotical stability of the closed-loop system. Note that the semi-global practical stability of the problem in question is a special case of that in Theorem 1. As such, we mainly focus on the development of the locally asymptotical stability.

Let $\sigma_{1}=\max \left\{\left\|\left[x^{T}, \zeta^{T}\right]^{T}\right\| \mid(x, \zeta) \in \Omega_{\rho}\right\}$ and $\sigma=$ $\min \left\{\sigma_{1}, \varepsilon\right\}$. It follows from Assumption 2 and Lemma 4 that there exist some positive numbers $b_{0}, k_{0}$, and a $\mathcal{C}^{1}$ function $V_{\sigma}(x)$ satisfies

$$
\frac{\partial V_{\sigma}}{\partial x} f_{c}(x) \leq-b_{0}\|x\|^{2},\left\|\frac{\partial V_{\sigma}}{\partial x}\right\| \leq k_{0}\|x\|,
$$

for all $x \in \bar{Q}_{\sigma}^{n} \triangleq\left\{x \in \mathbb{R}^{n}:\|x\| \leq \sigma\right\}$.

Since $\omega(t)=0$, by Assumptions 1 and 2, we can conclude that $\psi_{1}(x, \zeta)$ is continuously differentiable and satisfies $\psi_{1}(0,0)=0$. Thus there exist positive constants $k_{1}>0$ and $k_{2}>0$ such that

$$
\begin{array}{r}
\left\|\psi_{1}(x, \zeta)\right\|=\left\|\int_{0}^{x} \frac{\partial \psi_{1}(s, \zeta)}{\partial s} \mathrm{~d} s\right\| \leq k_{1}\|x\|, \\
\psi_{3}(x, \zeta)\left(d_{\Xi}(x, \zeta)-\operatorname{SAT}_{M}\left(d_{\Xi}(x, \zeta)-\zeta\right)\right) \leq k_{2}|\zeta| .
\end{array}
$$

for $(x, \zeta) \in \bar{Q}_{\sigma}^{n} \times \bar{Q}_{\sigma}$ and $\omega \in \mathcal{D}$. By inequalities (20), (33), (34) and Lemma 1 , for $(x, \zeta) \in \bar{Q}_{\sigma}^{n} \times \bar{Q}_{\sigma}$, one can obtain the derivatives of $V_{\sigma}(x)$ and $U(\zeta)$ along the closed-loop system (18) as

$$
\begin{aligned}
\dot{V}_{\sigma}(x) & \leq-b_{0}\|x\|^{2}+2 k_{0} \nu_{1}\|x\||\zeta| \\
& \leq-\frac{b_{0}}{2}\|x\|^{2}+\frac{2 k_{0}^{2} \nu_{1}^{2}}{b_{0}}|\zeta|^{2},
\end{aligned}
$$

and

$$
\begin{aligned}
\dot{U}(\zeta) & \leq-L|\zeta|^{2}+k_{1}\|x\||\zeta|+k_{2}|\zeta|^{2} \\
& \leq-L|\zeta|^{2}+\frac{b_{0}}{4}\|x\|^{2}+\left(\frac{k_{1}^{2}}{b_{0}}+k_{2}\right)|\zeta|^{2}
\end{aligned}
$$

where $\nu_{1}$ is a positive constant. Define a composite Lyapunov function $W_{\sigma}(x, \zeta)=V_{\sigma}(x)+U(x, \zeta)$ for the closed-loop system (18), whose derivative is obtained from (35) and (36), and given by

$$
\dot{W}_{\sigma}(x, \zeta) \leq-\frac{b_{0}}{4}\|x\|^{2}-\left(L-k_{2}-\frac{k_{1}^{2}}{b_{0}}-\frac{2 k_{0}^{2} \nu_{1}^{2}}{b_{0}}\right)|\zeta|^{2},
$$

for $(x, \zeta) \in \bar{Q}_{\sigma}^{n} \times \bar{Q}_{\sigma}$. Clearly, by selecting $L>$ $\max \left\{L^{*},\left(k_{2} b_{0}+k_{1}^{2}+2 k_{0}^{2} \nu_{1}^{2}\right) / b_{0}\right\}$, the locally asymptotical stability of the closed-loop system is guaranteed in the local region $(x, \zeta) \in \bar{Q}_{\sigma}^{n} \times \bar{Q}_{\sigma}$.

To conclude, in the presence of nonlinear uncertainties, the proposed control approach renders the asymptotical stability of the closed-loop system with a basin of attraction that could be arbitrarily large.

Remark 3: It is worthy noting that the asymptotic stability for the nonlinear systems with constant disturbances in Theorem 2 can also be achieved in a similar way, where the detailed proof is omitted for space. 


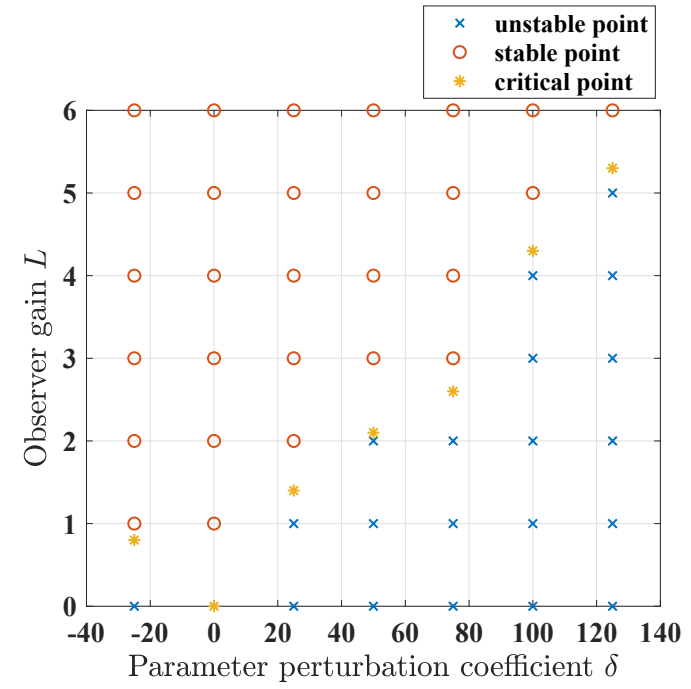

Fig. 1. Diagram of stability region in terms of the observer gain factor $L$ and parameter perturbation coefficient $\delta$.

\section{A Single-link Manipulator Example}

To validate the statements claimed previously, the control problem for a single-link manipulator systems is considered in this section. The dynamic model is described, which is given by [22]

$$
\begin{aligned}
\mathcal{M} \ddot{q}+\mathcal{B} \dot{q}+\mathcal{N} \sin (q) & =I \\
\mathcal{D} \dot{I}+\mathcal{H} I & =V-k_{m} \dot{q},
\end{aligned}
$$

where $q, \dot{q}$ and $\ddot{q}$ denote the link angular position, velocity, and acceleration, respectively. $I$ denotes the motor armature current, and $V$ is the voltage. $\mathcal{D}=\mathcal{D}_{0}+\delta \mathcal{D}_{0}$ is the uncertain parameter, where $\mathcal{D}_{0}$ denotes the nominal value of $\mathcal{D}$ and $\delta$ is a parameter perturbation coefficient sketching the uncertainty of parameter $\mathcal{D}$. The value of parameters are given as $\mathcal{M}=1, \mathcal{D}_{0}=0.5, \mathcal{B}=1, k_{m}=10, \mathcal{H}=0.5$, and $\mathcal{N}=10$. Let $x_{1}=q, x_{2}=\dot{q}, x_{3}=I$, and $u=V$. A single-link manipulator system is expressed as

$$
\begin{aligned}
& \dot{x}_{1}=x_{2}, \\
& \dot{x}_{2}=\frac{1}{\mathcal{M}} x_{3}+f_{2}(x), \\
& \dot{x}_{3}=\frac{1}{\mathcal{D}_{0}} u+f_{3}(x, u),
\end{aligned}
$$

where $f_{2}(x)=-\frac{\mathcal{N}}{\mathcal{M}} \sin \left(x_{1}\right)-\frac{\mathcal{B}}{\mathcal{M}} x_{2}, f_{3}(x, u)=-\frac{k_{m}}{\mathcal{D}_{0}} x_{2}-$ $\frac{\mathcal{H}}{\mathcal{D}_{0}} x_{3}-\frac{\mathcal{D}-\mathcal{D}_{0}}{\mathcal{D}_{0}}\left(\frac{1}{\mathcal{D}} u-\frac{k_{m}}{\mathcal{D}} x_{2}-\frac{\mathcal{H}}{\mathcal{D}} x_{3}\right)$.

To this end, the lumped uncertainty of the system (38) is collected and given by

$$
d(x, u)=-\frac{\mathcal{D}-\mathcal{D}_{0}}{\mathcal{D}_{0}}\left(\frac{1}{\mathcal{D}} u-\frac{k_{m}}{\mathcal{D}} x_{2}-\frac{\mathcal{H}}{\mathcal{D}} x_{3}\right) .
$$

Assume that the measurable states of the system with measurement noises are expressed as:

$$
\begin{aligned}
& y_{1}=x_{1}+\varsigma_{1}, \\
& y_{2}=x_{2}+\varsigma_{2}, \\
& y_{3}=x_{3}+\varsigma_{3},
\end{aligned}
$$
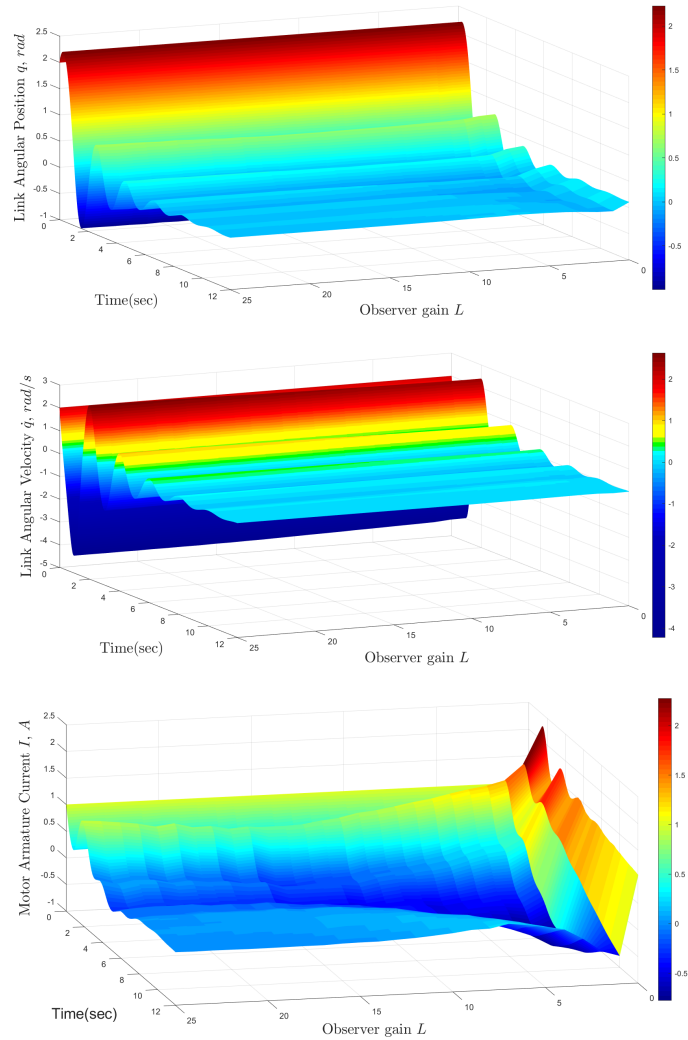

Fig. 2. System state $x$ for different observer gain factor $L$.
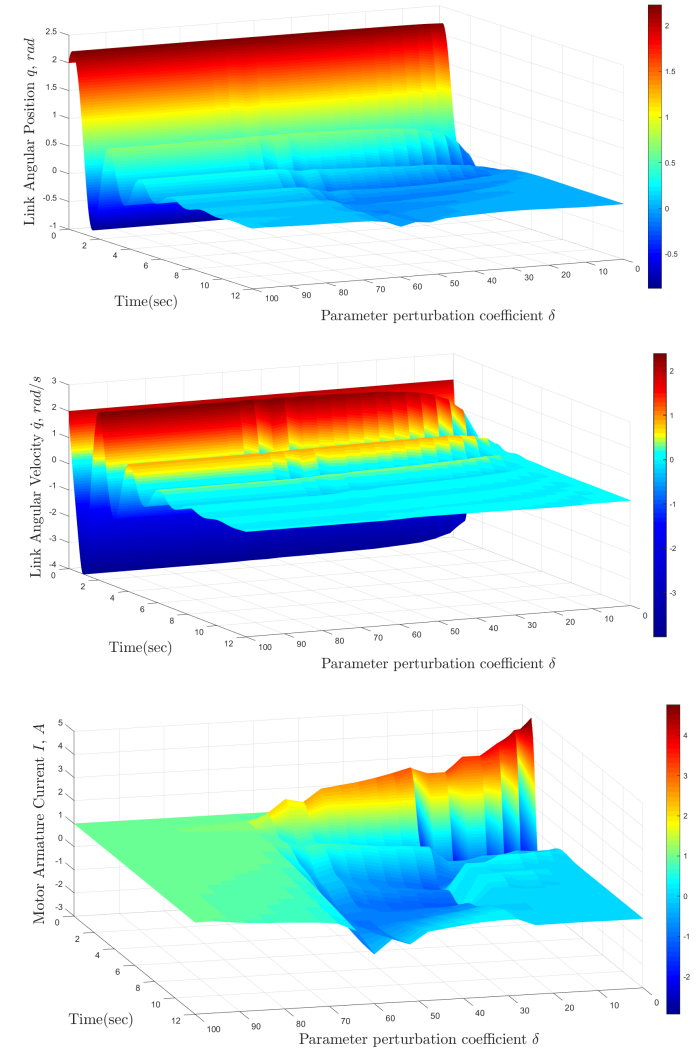

Fig. 3. System state $x$ for different parameter perturbation coefficient $\delta$. 
where $\varsigma_{i}(i=1,2,3)$ are measurement noises and obey norm distribution in $3 \sigma$ rules (i.e. $\varsigma_{i} \sim N\left(0, \frac{1}{9}\right)$ ). By applying the proposed control method in this paper, the disturbance estimation and the control law are designed as

$$
\begin{aligned}
& \dot{\xi}=-L\left(-\frac{k_{m}}{\mathcal{D}_{0}} y_{2}-\frac{\mathcal{H}}{\mathcal{D}_{0}} y_{3}+\frac{1}{\mathcal{D}_{0}} u+\hat{d}\right), \\
& u=\mathcal{D}_{0}\left(-k_{1} y_{1}-k_{2} y_{2}-k_{3} y_{3}-\operatorname{SAT}_{M}(\hat{d})\right),
\end{aligned}
$$

where $k_{1}, k_{2}$ and $k_{3}$ are the feedback control gains, and $\hat{d}$ is the estimate of the lumped uncertainty generated by the nonlinearity estimator (8).

For simulation studies, the controller gains in (39) are chosen as $k_{1}=2, k_{2}=1.5, k_{3}=1$. The initial state values are taken as $x=[2,2,1]^{T}$. Since this paper mainly focuses on uncertainty estimation and compensation, we assume that there is no external disturbance during the simulation. The threshold of the saturation function is designed as $M=50$.

To evaluate the qualitative robustness of the propose method, we focus on investigating the relationship between the observer gain factor $L$ and parameter perturbation coefficient $\delta$ on the stability of the system (37). In the simulation, $\delta$ is supposed to range from -25 to 125 , whereas the observer gain $L$ is assumed to range from 0 to 6 . The diagram of stability region of the system in terms of the observer gain factor $L$ and parameter perturbation coefficient $\delta$ is shown in Fig. 1. It has been clearly shown by Fig. 1 that the observer gain factor $L$ should be substantially increased to render stability as the increase of uncertainty parameter perturbation coefficient $\delta$. We further conduct two cases of simulations to qualitatively demonstrate the robust stability of the presented control approach. For the first one, the parameter perturbation coefficient $\delta$ is fixed as $\delta=120$, and the tracking control performance under different observer gain is investigated. The results in this case are shown in Fig. 2, which shows that the closed-loop system tends to be unstable as the observer gain $L$ decreases to about 5 . For the second one, the robust stability of the controller with fixed observer gain $L=2$ is investigated, where the result is shown by Fig. 3. It can be observed from the figure that the stability as well as control performance is getting worse with the increase of the parameter perturbation coefficient $\delta$.

\section{Conclusion}

A nonlinearity estimator-based control approach has been proposed in this paper to address the robust control problem of a class of nonlinear systems subject to both disturbances and uncertainties. The qualitative robustness stability has been established for the closed-loop system in the sense of semiglobal practical and asymptotical stabilization. The results obtained have provided theoretical justifications for DUEA approaches to estimate and compensate nonlinearities for nonlinear systems in practice. The simulation results on a singlelink manipulator have demonstrated the qualitative relationship between observer gain factor and quantity of nonlinearities.

\section{ACKNOWLEDGEMENT}

The authors would like to express their sincere thanks to Professor Alberto Isidori at the University of Rome for his constructive suggestions on semi-global stability analysis tools which are used in this paper.

\section{APPENDIX: PROOF OF LEMMA 2}

\section{REFERENCES}

[1] H. Khalil, Nonlinear Systems. New Jersey: Prentice Hall, 2002.

[2] A. Isidori, Nonlinear Control Systems II. New York: SpringerVerlag, 1999.

[3] A. Isidori, Lectures in Feedback Design for Multivariable Systems. Switzerland: Springer, 2016.

[4] K. Zhou, and J.C. Doyle, Essentials of Robust Control. Upper Saddle River, NJ: Prentice-Hall, 1998.

[5] W.-H. Chen, J. Yang, and Z. Zhao, "Robust control of uncertain nonlinear systems: a nonlinear DOBC approach," Journal of Dynamic Systems, Measurement, and Control, 138(7), 071002, 2016.

[6] C.D. Johnson, "Optimal control of the linear regulator with constant disturbances," IEEE Transactions on Automatic Control, 13(4), 416-421, 1968.

[7] K. Ohishi, M. Nakao, K. Ohnishi, and K. Miyachi, "Microprocessorcontrolled dc motor for load-insensitive position servo system," IEEE Transactions on Industrial Electronics, 34(1), 44-49, 1987.

[8] J. Han, "Extended state observer for a class of uncertain plants," in Chinese, Control Decision, 10(1), 85-88, 1995.

[9] X.J. Wei, Z.J. Wu, and H.R. Karimi, "Disturbance observerbased disturbance attenuation control for a class of stochastic systems," Automatica, 63, 21-25, 2016.

[10] M. Chen, P. Shi, and C.C. Lim, "Robust constrained control for MIMO nonlinear systems based on disturbance observer," IEEE Transactions on Automatic Control, 60(12), 3281-3286, 2015.

[11] W. Xue, and Y, Huang, "Performance analysis of 2-DOF tracking control for a class of nonlinear uncertain systems with discontinuous disturbances," International Journal of Robust and nonlinear Control, 28, 1456-1473, 2018.

[12] A. Chakrabortty and M. Arcak "Time-scale separation redesigns for robust stabilization and performance recovery of uncertain nonlinear systems", Automatica, 45(1), 34-44, 2009.

[13] L. Praly and Z.-P. Jiang "Semiglobal stabilization in the presence of minimum-phase dynamic input uncertainties", in the Proceedings of the IFAC NOLCOS, 1998, pp.321-326.

[14] L.B. Freidovich, and H.K. Khalil, "Performance recovery of feedback-linearization-based designs," IEEE Transactions on Automatic Control, 53(10), 2324-2334, 2008.

[15] J. Back, and H. Shim, "An inner-loop controller guaranteeing robust transient performance for uncertain MIMO nonlinear systems," IEEE Transactions on Automatic Control, 54(7), 1601-1607, 2009.

[16] W.-H. Chen, D.J. Ballance, P.J. Gawthrop, and J. O'Reilly, "A nonlinear disturbance observer for robotic manipulators," IEEE Transactions on industrial Electronics, 47(4), 932-938, 2000.

[17] B.G. Guo, and Z.L. Zhao, "On the convergence of an extended state observer for nonlinear systems with uncertainty," Systems and Control Letters, 60(6), 420-430, 2011.

[18] W. Xue, and Y. Huang, "On performance analysis of ADRC for a class of MIMO lower-triangular nonlinear uncertain systems," ISA Transactions, 53(4), 955-962, 2014. 
[19] T. Jiang, C. Huang, and L. Guo, "Control of uncertain nonlinear systems based on observers and estimators," Automatica, 59, 35-47, 2015.

[20] A. Teel, and L. Praly, "Tools for semiglobal stabilization by partial state and output feedback," SIAM Journal on Control and Optimization, 33, 1443-1488, 1995.

[21] B. Yang, and W. Lin, "Semi-global stabilization of nonlinear systems by nonsmooth output feedback," International Journal of Robust and Nonlinear Control, 24, 2522-2545, 2014.

[22] H. Sun and L. Guo, "Neural network-based DOBC for a class of nonlinear systems with unmatched disturbances," IEEE Transactions on Neural Networks and Learning Systems, 28, 482-489, 2017. 